\title{
Molecular mechanism of SARS-CoV-2 components caused ARDS in murine model
}

\author{
Tingxuan $\mathrm{Gu}^{1,2^{*}}$, Simin $\mathrm{Zhao}^{2,4^{*}}$, Guoguo $\mathrm{Jin}^{2,5}$, Mengqiu Song ${ }^{1,2}$, Yafei Zhi ${ }^{2}$, Ran Zhao ${ }^{1,2}$, Fayang \\ $\mathrm{Ma}^{1,2}$, Yaqiu Zheng ${ }^{2}, \mathrm{Keke} \mathrm{Wang}^{2}$, Hui Liu ${ }^{1,2}$, Mingxia Xin ${ }^{2}$, Wei Han ${ }^{2}, \mathrm{Xiang} \mathrm{Li}^{1,2,3}$, Christopher D \\ Dong $^{6}$, Kangdong Liu ${ }^{1,2,3}$, Zigang Dong ${ }^{1,2+}$ \\ ${ }^{1}$ Department of Pathophysiology, School of Basic Medical Sciences, Academy of Medical Science, \\ College of Medicine, Zhengzhou University, Zhengzhou, Henan, P.R. China \\ ${ }^{2}$ China-US (Henan) Hormel Cancer Institute, Zhengzhou, Henan, P.R. China \\ ${ }^{3}$ Henan Provincial Cooperative Innovation Center for Cancer Chemoprevention, Zhengzhou University, \\ Zhengzhou, Henan, P.R. China \\ ${ }^{4}$ Affiliated Cancer Hospital of Zhengzhou University, Zhengzhou, Henan, P.R. China \\ ${ }^{5}$ The Henan Luoyang Orthopedic Hospital, Zhengzhou, Henan, P.R. China \\ ${ }^{6}$ Wartburg College, Waverly, IA, USA \\ "These authors contributed equally to this work
}

Running Title: Constructed a mimic murine model for COVID-19

\section{Corresponding Author :}

Professor Zigang Dong,

Department of Pathophysiology, School of Basic Medical Sciences, Academy of Medical Science,

College of Medicine, Zhengzhou University, Zhengzhou, Henan, China

Fax) +86-371-65587670; Phone) +86-371-65587008; E-mail)dongzg@zzu.edu.cn 
Abstract: COVID-19 has become a major challenge to global health, and until now, no efficient antiviral agents have been developed. The SARS-CoV-2 infection is characterized by pulmonary and systemic inflammation in severe patients, and acute respiratory distress syndrome (ARDS) caused respiratory failure contributes to most mortalities. There is an urgent need for developing effective drugs and vaccines against SARS-CoV-2 and COVID-19 caused ARDS. However, most researchers cannot perform SARS-CoV-2 related researches due to lacking P3 or P4 facility. We developed a non-infectious, highly safety, time-saving SARS-CoV-2 components induced murine model to study the SARS-CoV-2 caused ARDS and cytokine storm syndrome (CSS). We also investigated mAbs and inhibitors which potentially neutralize the pro-inflammatory phenotype of COVID-19, and found that anti-IL-1 $\alpha$, anti-IL-6, anti-TNF $\alpha$, anti-GM-CSF mAbs, p38 inhibitor, and JAK inhibitor partially relieved CSS. Besides, anti-IL-6, anti-TNFa, anti-GM-CSF mAbs and inhibitors of p38, ERK, and MPO somewhat reduced neutrophilic alveolitis in the lung. In all, we established the murine model mimic of COVID-19, opening a biosafety and less time-consuming avenue for clarifying the mechanism of ARDS and CSS in COVID-19 and developing the therapeutic drugs.

Keywords: COVID-19, SARS-CoV-2, Acute respiratory distress syndrome, Cytokine storm syndrome, Murine model 


\section{Introduction}

In early December 2019, pneumonia of an unknown cause appeared in Wuhan. The virus strain was first isolated from patient samples on January 7, 2020, and the whole genome sequence of the virus was then obtained on January $10,2019^{1-3}$. The genome of the virus was compared with SARS-CoV and MERS-CoV, and the homology was about $79 \%$ and 50\% respectively. Therefore, it was named as novel coronavirus (SARS-CoV-2) ${ }^{4}$. COVID-19 is caused by SARS-CoV-2 infection ${ }^{5}$. A total of 7,410,510 confirmed cases of COVID-19 caused by SARS-CoV-2 have been reported globally as of June $14^{\text {th, }}$ 2020. The total number of deaths worldwide reached 418,294. Until now, no specific antiviral drugs or vaccines for SARS-CoV-2 have been developed. The epidemic of COVID-19 is threatening public health. Methods to control the virus and improve the treatments have quickly become urgent issues concerning national security across the world.

As the COVID-19 progressing, patients may develop acute respiratory distress syndrome (ARDS), cytokine storm syndrome $(\mathrm{CSS})^{6}$. CSS, which showed a disorder of the immune system and a rapid increase of pro-inflammatory cytokine levels after the stimulus, has been reported to associate with the deterioration of various severe diseases ${ }^{7}$. In COVID-19 patients, the CSS occurred when the plasma levels of pro-inflammatory cytokines as IL-6, IL-1 $\beta$, IL-2, IL-8, IL-17, G-CSF, GM-CSF, IP10, MCP1, MIP1 $\alpha$ and TNF $\alpha$ were significantly increased, recruiting immune cells through a positive feedback loop, eventually forming a cytokine $\operatorname{storm}^{8-10}$. Therefore, clarifying the mechanism of CSS in COVID-19 patients and discovering the key cytokines contribute to developing more effective methods to block CSS, which is of great significance for the treatment of COVID-19 patients. The continued global epidemic of COVID-19 emphasizes the necessity of establishing a COVID-19 related pneumonia animal model. Currently, most animal models have used virus strains isolated from 
COVID-19 patients, which can simulate human pathophysiological processes, but it lacks biosecurity and can only be performed in the biosecurity $\mathrm{P} 3 / \mathrm{P} 4 \operatorname{labs}^{11,12}$. Therefore, there is an urgent need to establish an effective animal model for the research of COVID-19 pneumonia that is non-infectious and can be performed in less well-equipped laboratories.

Here, our laboratory has successfully developed a highly biosecurity and time-saving ARDS animal model, which simulates the CSS and the pathophysiological changes happened in the COVID-19 patients by using Poly I:C and SARS-CoV-2 spike protein (Poly I:C and SP). The combination of Poly I:C and SP were used as SARS-CoV-2 mimic. We identified some neutralizing mAbs and inhibitors have some prophylactic efficacy for COVID-19. More importantly, it could have useful implications for the intervention of current and afterward coronavirus infectious diseases.

\section{Results}

First, we inoculated SARS-CoV-2 mimic into mice lung through intratracheal injection (Figure 1a).

After the administration of Poly I:C and SP, acute lung injury featuring neutrophilic inflammation and interstitial edema were observed. The lung tissue showed severe injury with Poly I:C and SP, which was more severe than Poly I:C induced only. To better understand the time course of pathologic changes, we explored the time point of lung injury. The lung injury occurred at $6 \mathrm{~h}$, was most severe at $24 \mathrm{~h}$, but gradually decreased at $48 \mathrm{~h}$ (Figure 1b). Besides, the mice suffered from extensive pleural fluid accumulation between $24 \mathrm{~h}$ and $48 \mathrm{~h}$ post-challenge. Pleural effusion was measured by using small animal MRI. Gross lung lesions were observed at $24 \mathrm{~h}$ after challenge, reduced at $48 \mathrm{~h}$, and not apparent at $6 \mathrm{~h}$ (Figure 1c).

To understand the molecular pathology of Poly I:C and SP induced lung injury, cytokines and flow cytometric analysis were performed to determine the inflammatory molecules and related immune cells. 
The level of IL-1 $\alpha$, IL-6 and TNF $\alpha$ were dramatically increased in Poly I:C plus SP group as compared with Poly I:C or SP alone group. Saline and recombinant FC protein cannot stimulate the production of cytokines, Poly I:C or SP shows a higher level of IL-1 $\alpha$, IL-6, and TNF $\alpha$ (Figure 2a). Intratracheal injection of saline, SP and recombinant FC protein cannot stimulate an increase of dsDNA, an indicator of NETs (Figure 2b). Total cellularity of mice bronchoalveolar lavage (BAL) samples showed a significant increase in an SP dose-dependent manner after 24 h challenge (Figure 2c). Furthermore, investigation of cellularity in the mice BAL samples by flow cytometry. The result showed that neutrophils, but not macrophages, significantly infiltrated and migrated into the lungs. It should be noted that the number of neutrophils in the BAL at SP dose-dependent increased compared with saline (Figure 2d). For the time point study, the SARS-CoV-2 mimic led to an increase in the inflammatory cytokines IL-1 $\alpha$, IL-6 and TNF $\alpha$ at $6 \mathrm{~h}$. However, TNF $\alpha$ and IL-6 significantly decreased at $24 \mathrm{~h}$ timepoint as compare with $6 \mathrm{~h}$ timepoint. When compared with saline, all cytokines levels were increased at $24 \mathrm{~h}$ timepoint (Figure 2e). We noted that there was no significant difference in the infiltration of neutrophils between those two time points (Figure 2f). Moreover, a unique mechanism of the neutrophil effector is the generation of neutrophil extracellular traps (NETs); the level of NETs can be quantified by measuring cell-free double-stranded DNA (dsDNA). In our study, the concentration of dsDNA was used as an indicator of NETs. At the $6 \mathrm{~h}$ and $24 \mathrm{~h}$ time point after challenge, there was no significant difference in the concentration of dsDNA in the mice BAL samples (Figure $2 \mathrm{~g}$ ).

Furthermore, we dissociated cells from the mice lung tissue after $24 \mathrm{~h}$ installation of Poly I:C and SP, and maintained for $6 \mathrm{~h}$ and $24 \mathrm{~h}$. The concentrations of IL- 6 were still increasing after dissociation, and longer maintaining produced more IL-6 into the medium. This indicated that CSS happens in the lungs 
after installation of SARS-CoV-2, along with immune cells such as tissue-resident macrophages highly inflammatory and activated (Figure $2 \mathrm{~h}$ ).

We hypothesized that one or more cytokines drove the CSS. To validate the hypothesis, we applied the neutralizing antibody such as anti-IL-1 $\alpha$, anti-IL-6 or anti-TNF $\alpha$ and blocking antibody anti-IL6R or anti-TNFR2 in this animal model. Neutralizing IL-6 reduced the production of cytokine IL-6 in BAL, with no effect on IL-1 $\alpha$ and TNF $\alpha$ (Figure 3a). However, IL-6R blocking mAbs was unable to alter the concentrations of IL-1 $\alpha$, IL-6 and TNF $\alpha$ in BAL (Figure 3b). Anti-TNF $\alpha$ mAbs reduced the production of cytokine TNF $\alpha$ in BAL, with no effect on IL-1 $\alpha$ and IL-6 (Figure 3c). However, TNFR2 mAbs failed to alter the production of IL-1 $\alpha$, IL-6 and TNF $\alpha$ (Figure 3d). Neutralizing mAbs targeting IL-1 $\alpha$ directly reduced the cytokines IL-1 $\alpha$, and IL-6 also reduced significantly, but not TNF $\alpha$ (Figure 3e). In addition, concentrations of GM-CSF not increased in BAL during SARS-CoV-2 challenge, but anti-GM-CSF mAbs could reduce the production of TNFa (Figure 3f).

Infiltration of neutrophils caused the neutrophilic alveolitis during SARS-CoV-2 challenge. The percent of neutrophils in BAL were tested after anti-IL-6 (Figure 3g), anti-IL6R (Figure 3h), anti-TNFa (Figure 3i), anti-TNFR2 (Figure 3j), anti-IL-1 $\alpha$ (Figure 3k) and anti-GM-CSF (Figure 31) treatment. The result indicated that neutralizing of cytokines IL-6, TNF $\alpha$ and GM-CSF or blocking the IL-6R could reduce the infiltration of neutrophils. Concentrations of dsDNA were no reduction after antibodies treatment (Figure 3m). Next, we treated RAW264.7 cells with Poly I:C and evaluated the production of IL- 6 in the medium. The results indicated a reduced trend of IL-6 while treating with 20 $\mu \mathrm{g}$ antibodies although not significantly (Figure 3n). Our histological analysis results indicated that treatment with neutralizing or blocking mAbs of inflammatory cytokines could partially reduce the 
neutrophilic inflammation and interstitial edema in the lung, but not anti-TNFR2 (Figure 3o). Therefore,

potent therapeutic efficacy in a synergistic manner can be considered in clinical.

NETs can also be generated by macrophages ${ }^{13}$, and strongly contribute to acute lung injury during virus infection ${ }^{14-16}$. We hypothesized that the SARS-CoV-2 mimic stimulates the macrophages and produce inflammatory cytokines, such as IL-1 $\alpha$, IL-6 and TNF- $\alpha$, recruit and activate neutrophils. P38 and ERK are the signaling mediators of NETs formation. MPO and Elastase as the peroxidase enzyme in neutrophils and essential for NETs formation ${ }^{17,18}$. TLR3/dsRNA complex inhibitor can block the interactions between TLR3 and Poly I:C. JAK inhibitor can block the downstream signaling of IL-6. In our study, we found that the inhibition of P38 reduces IL-1 $\alpha$ and inhibition of JAK reduces IL-6 significantly (Figure 4a). We noticed a decrease in the infiltration of neutrophils after treatment of P38, ERK and MPO inhibitors, which all targeted activities of neutrophils (Figure 4b). There was no significant difference in the concentrations of dsDNA after the treatment of those inhibitors (Figure 4c). Furthermore, we performed the cell assay to validate the effect of TLR3/dsRNA inhibitor and JAK inhibitors on mouse primary macrophages. TLR3/dsRNA inhibitor did not reduce the IL-6 production at $45 \mu \mathrm{M}$. JAK1/2 inhibitor Baricitinib reduced the production of IL-6 significantly, but not Febratinib (JAK2 inhibitor) (Figure 4d). Histological analysis result indicated that inhibitors of P38, ERK, MPO and Baricitinib (JAK inhibitor) obviously decreased the neutrophilic inflammation and interstitial edema in the lung (Figure 4e).

\section{Discussion}

SARS-CoV-2 has posed a serious threat to global public health. Antiviral drugs, such as hydroxychloroquine, chloroquine and remdesivir did not achieve an ideal therapeutic effect ${ }^{19,20}$. In order to understand the molecular mechanism of SARS-CoV-2 induced ARDS/CSS and find an effective 
strategy to prevent and remedy this highly infectious disease, we have developed a murine ARDS

model, to mimic pathological changes in COVID-19 patient. Coronavirus includes a large genomic

RNA and can stimulate TLR3/7 upon infection ${ }^{21-25}$. We used Poly I:C to mimic the effect of viral RNA.

Moreover, the unique outcome of recombinant SP was showed in a TNF $\alpha$ outbreak featured CSS when

compared with Poly I:C challenged mice. The TNF $\alpha$ storm revealed that the underlying mechanism of

CSS happened in COVID-19 patients, not just IL-6 or IL-1 $\alpha$, TNF $\alpha$ may play a more critical role. As a

new target in our model, anti-TNF $\alpha$ may need a tight treatment window, the TNF $\alpha$ burst was observed

after $6 \mathrm{~h}$ administration of SARS-Cov-2 mimic and disappeared at $24 \mathrm{~h}$. Furthermore, IL-6 maintained

its high level during the administration of SARS-CoV-2 mimics, anti-IL-6, or related signaling may

provide a relatively broad treatment window.

Impaired inflammatory responses in severe COVID-19 patients provide a distinct pattern of

COVID-19 progression in host immune response to the SARS-CoV-2 infection, with exhausted

lymphocytes, high neutrophil to lymphocyte ratio, CSS in severe cases of COVID-19,26-29. CSS was

found to be the major cause of morbidity in patients with SARS-CoV and MERS-CoV ${ }^{30}$, with the

presence of IL-6 in the plasma being a hallmark of severe infections. Clinical trials using IL-6 and

IL-6R antagonists are underway ${ }^{31}$. In our research, we first tested the effect of IL-6 or IL-6R mAbs,

IL-6 in BAL was affected, but IL-1 $\alpha$ and TNF $\alpha$ no changes. Importantly, treatment of IL-6 or IL-6R

mAbs reduced neutrophil infiltration. This result may indicate that blocking IL-6 signaling shows

partially benefit from the SARS-CoV-2 mimics induced neutrophilic alveolitis, and CSS in lung did not

appear to effective mitigation. Furthermore, blocking of JAK-STAT3 signaling also could inhibit the

IL-6 mediated signaling transduction. For blocking TNF $\alpha$ signaling, we performed the TNF $\alpha$ and

TNFR2 mAbs. TNF exerts its effect by stimulation of two different receptors, TNFR1 and TNFR2. 
TNFR1 is expressed in all cell types, but TNFR2 is only expressed in certain cell types, such as myeloid cells, glial cells and T and B cell subsets. Neutralizing TNF $\alpha$ via mAbs reduced the production of TNF $\alpha$ in our model, IL-6 and IL-1 $\alpha$ not affected. Treatment of TNF $\alpha$ mAbs, but not TNFR2 mabs reduced neutrophil infiltration. Those results indicated that TNF $\alpha$ signaling shows partially reduced neutrophilic alveolitis and benefit from SARS-Cov-2 mimic induced CSS. GM-CSF is a cytokine found in high levels in patients with COVID-19, anti-GM-CSF mAbs was used for the treatment of COVID-19 clinical trial (NCT04400929) $)^{2}$. In our present data, anti-GM-CSF reduced neutrophil infiltration and decreased the TNF $\alpha$ during SARS-Cov-2 mimic challenge. Even we did not find GM-CSF enriched in BAL, but anti-GM-CSF treatment shows protection effect from neutrophilic alveolitis and NETs induced lung injury.

It was reported that macrophages in the lungs may contribute to inflammation by producing multiple cytokines/chemokines and recruiting more inflammatory monocytic cells and neutrophils ${ }^{32,33}$. We have applied various signal pathway inhibitors to block the dysregulated macrophages and neutrophils.

TLR3/dsRNA Complex inhibitor blocked the dsRNA and TLR3 interaction, but in our present data,

TNF $\alpha$ in BAL cannot induced by single Poly I: C. and SARS-Cov-2 includes a single strand RNA genome, TLR7 also sense the viral RNA ${ }^{34}$.

To prevent sustained activation of neutrophils, we applied P38 inhibitor SB203580, ERK inhibitor Tauroursodeoxycholate and MPO inhibitor 4-Aminobenzoic hydrazide in the SARS-CoV-2 mimic model. It was reported that MPO is a functional and activation marker of neutrophils. Elastase was produced by neutrophils and can mediate lung injury. Inhibitors of MPO and elastase can directly reduce the acute lung injury at the inflammatory site ${ }^{18,35,36}$. In our study, inhibitors include P38 inhibitor, ERK inhibitor, MPO inhibitor and elastase inhibitor can reduce the neutrophil infiltration into 
lung, but not elastase. Here we used the neutralizing or blocking antibodies, essential pathway

inhibitors of the innate and adaptive immune responses to target the most abundant cytokines, NETs

and other pro-inflammatory factors to prevent the SARS-CoV-2 caused CSS and ARDS. Our data

indicate that potential combination of those neutralizing/blocking antibodies or inhibitors may

contribute to the treatment of CSS and ARDS, and should be investigated further.

Taken together, we established a non-infectious, high safety and time-saving murine robustness model

by using Poly I:C and SP, which mimic the pathological changes of SARS-CoV-2 caused CSS and

ARDS. This model can be available for research on COVID-19 caused CSS and ARDS, addressing the immunopathology alterations, and exploring new therapies of COVID-19.

\section{Methods and material}

\section{Experimental Animals}

Male BALB/c mice (8-10 weeks) free of pathogens were purchased from Beijing Vital River Laboratory Animal Technology Co., Ltd. (Beijing, China). Mice were housed in a pathogen-free environment under conditions of $20^{\circ} \mathrm{C} \pm 2{ }^{\circ} \mathrm{C}, 50 \% \pm 10 \%$ relative humidity, 12 -h light/dark cycles. They were provided with food and water ad libitum. All experimental procedures involving animals were approved by the Ethics Review Commission of Zhengzhou University (following internationally established guidelines).

\section{COVID-19 ARDS murine model and treatment}

Mice were anesthetized via intraperitoneal (IP) injection with Pentobarbital Sodium (50 mg/kg). A small incision was made over the trachea, and the underlying muscle and glands were separated to 
expose the trachea. Mice were intratracheally administered with freshly mixed Poly I:C (Poly

I:C-HMW, Invivogen, tlrl-pic) $2.5 \mathrm{mg} / \mathrm{ml}$ and SARS-CoV-2 recombinant spike protein (ECD-His-tag,

Genescript, Z03481) $15 \mu \mathrm{g}$ (in saline), followed by $100 \mu \mathrm{L}$ air. 2.5 mg/kg Poly I:C, FC control (ACRO,

P01857-1), $15 \mu \mathrm{g}$ SARS-CoV-2 recombinant spike protein and saline were administered intratracheally independent at the same volume as control.

Blocking and neutralizing antibodies anti-IL-1 $\alpha$ (InVivoMab, BE0243), IL-6R (InVivoMab, BE0047), and TNFR2 (InVivoMab, BE0247) were administrated intraperitoneally as a single dose of $200 \square \mu \mathrm{g}$ $24 \square \mathrm{h}$ in prior. TLR3/dsRNA inhibitor (Merck, 614310) was administrated via i.p. at $50 \mathrm{mg} / \mathrm{kg} 2 \mathrm{~h}$ prior to administration of SARS-CoV-2 mimics. MPO inhibitor (Merck, A41909) was administrated via i.p. at $50 \mathrm{mg} / \mathrm{kg}$ per day, $3 \mathrm{~d}$ prior to administration of SARS-CoV-2 mimics. P38 inhibitor (MCE, HY-10256) was administrated via i.p. at $20 \mathrm{mg} / \mathrm{kg}$ per day, $3 \mathrm{~d}$ prior to administration of SARS-CoV-2 mimics. ERK inhibitor (MCE, HY-19696A) was administrated via i.p. at $100 \mathrm{mg} / \mathrm{kg}$ per day, 3 d prior to installation of SARS-CoV-2 mimics. Elastase Inhibitor (GLPBIO, GC11981) was administrated via i.p. at $5 \mathrm{mg} / \mathrm{kg}$ per day, $3 \mathrm{~d}$ prior to administration of SARS-CoV-2 mimics. JAK inhibitor (MCE, HY-15315) was administrated via i.p. at $20 \mathrm{mg} / \mathrm{kg}$ per day, $3 \mathrm{~d}$ prior to administration of SARS-CoV-2 mimics.

\section{Analysis of BAL samples}

$6 \mathrm{~h}$ after the administration, mice were anesthetized via intraperitoneal (IP) injection of Pentobarbital

Sodium $50 \mathrm{mg} / \mathrm{kg}$. A $26 \mathrm{G}$ venous indwelling needle hose was inserted into the exposed tracheal lumen, and then the airway was washed three times with $1 \mathrm{ml}$ saline each, the first lavage fluid sample was kept separately to test cytokines.

\section{Multiplex cytokines assay}


BAL was collected from mice lung after anesthetized, the mice BAL were separated by centrifuging and stored at $-80 \square$. BAL sample collection was performed as previously described. Next, concentrations of mice inflammatory related cytokines IL-1 $\alpha$, IL-1 $\beta$, IL-6, IL-10, IL-12p70, IL-17A, IL-23, IL-27, MCP-1, IFN- $\beta$, IFN- $\gamma$, TNF- $\alpha$, and GM-CSF were measured by LEGENDplex ${ }^{\mathrm{TM}}$ Mouse Inflammation Panel (13-plex, Biolegend), the datas were harvest by flow cytometry using

FACSCalibur (BD).

\section{Flow Cytometry analysis}

Fluorescent labeled antibodies were performed to quantify neutrophils and macrophages. Unconjugated anti-mouse CD16/CD32 (Biolegend, 101320) was used for blocking Fc receptors, APC labeled anti-mouse Ly-6G/Ly-6C (Gr-1) and FITC labeled anti-mouse F4/80 were performed for neutrophils and macrophages, incubated $30 \mathrm{~min}$ on ice, protect from light. Washed three times by PBS and centrifuged to remove the supernatant and responded in $150 \mu \mathrm{L}$ PBS. Samples were analyzed in the BD FACSCalibur (BD).

\section{Histopathology Analysis}

An independent experiment was performed for the histopathology analysis of pulmonary. The mouse lungs were removed intact and weighted, then fixed in $10 \%$ formalin and paraffin-embedded. Three different fields from a lung section were evaluated, $3 \mu \mathrm{m}$ sections were sliced on a Leica model rotary microtome and stained with hematoxylin-eosin. Histological analysis was subjected by two independent skilled pathologists, in double-blind.

\section{Mouse macrophage cell culture}


RAW264.7 cell was cultured in DMEM medium supplemented with penicillin (100 units/mL), streptomycin $(100 \mu \mathrm{g} / \mathrm{mL})$, and 10\% FBS (Biological Industries, Kibbutz Beit-Haemek, Israel).

\section{Compounds treated to RAW264.7}

RAW264.7 cells $\left(6 \times 10^{5}\right.$ cells/well) were seeded into 12 -well plates and cultured for $24 \mathrm{~h}$ in incubator.

The cells were treated with a series dilution of compound X; meanwhile, Poly I:C $(10 \mu \mathrm{g} / \mathrm{ml})$ were added into medium in a 12-well plate. Meanwhile, RAW264.7 cell was subjected to DMEM with or without Poly I:C as a positive or negative control. The cells were maintained at $37^{\circ} \mathrm{C}$ in a $5 \% \mathrm{CO}_{2}$ incubator for $12 \mathrm{~h}$, harvested the supernatant for the detection of IL-6 secretion.

\section{Mouse primary macrophage cell culture}

Bone were gently separated from mouse, and the connecting muscles and soft-tissues were all removed from the bone. The macrophages contain in the bone were washed out by DPBS using $1 \mathrm{ml}$ syringe.

Suspended the eluate and centrifuged at $1000 \mathrm{rpm}$ for $5 \mathrm{~min}$. Washed the pellets by DPBS and resuspended the cell pellets by RPMI-1640 containing penicillin(100 units/mL), streptomycin (100 $\mu \mathrm{g} / \mathrm{mL}), 10 \%$ FBS (Biological Industries, Kibbutz Beit-Haemek, Israel) and 50 ng/ml M-CSF (peprotech), plated the cells in a low attachment culture dish and maintained at a $5 \% \mathrm{CO}_{2}$ incubator. Renew the medium of fresh RPMI-1640 complete medium contained $50 \mathrm{ng} / \mathrm{ml}$ M-CSF every other day until the density of adherent cells reached $90 \%$. Digested the cells and plated $6 \times 10^{5}$ cells/well into 12-well plates, cultured for $24 \mathrm{~h}$ and treated the Poly I:C and compound to the cell using blank

RPMI-1640 medium. This method is similar to the treatment of RAW264.7.

\section{Dissociated of primary lung cells mix}


Took out the whole lung tissues of the mice and washed by pre-cold PBS containing DNase I $(0.01$

$\mathrm{mg} / \mathrm{ml})($ Sigma), penicillin (100 units/mL), and streptomycin $(100 \mu \mathrm{g} / \mathrm{mL})$. Tattered the lung tissues

into small pieces in a sterile centrifuge tube by scissors, suspended the small pieces by $1 \mathrm{mg} / \mathrm{ml}$ Type I

collagenase (Sigma) supplied by $0.01 \mathrm{mg} / \mathrm{ml}$ DNase I and digested in a $37 \square$ shaking incubator for 30

min. Filtered the solution with a 200 mesh sieve and centrifuged at $1000 \mathrm{rpm}$ for $5 \mathrm{~min}$, subsequently.

After washed the pellets twice by pre-cold PBS containing DNase I $(0.01 \mathrm{mg} / \mathrm{ml})$, resuspended the

pellets by RPMI-1640 containing 10\% FBS (Biological Industries, Kibbutz Beit-Haemek, Israel) and

seeded into 12 -well plate. Harvested the supernatant after $24 \mathrm{~h}$ culture and removed the floating cells

by centrifuge at $3000 \mathrm{rpm}$ for $5 \mathrm{~min}$. The ELISA assay was performed to detect the IL- 6 concentration

following the manufacturer's instructions.

\section{Statistical Analysis}

Statistical analysis was performed using GraphPad Prism 7.0 (GraphPad Software, United States).

Specific statistical methods and comparisons made by methods as described in figure legend.

Comparison between two groups were performed by paired Student's t-test or unpaired Student's t-test.

$P<0.05$ was regarded as statistically significant and marked with a star, data was reported as

mean $\square \pm \square$ SEM, and error bars indicate SEM.

\section{Graphical Illustrations.}

Schematic illustrations were established with BioRender (BioRender.com).

Author contributions: Z.G.D., T.X.G. and S.M.Z. designed the study. T.X.G., S.M.Z., G.G.J, M.Q.S.,

Y.F.Z., R.Z., F.Y. M., Y.Q. Z., K.K.W. performed the main experiment. H.L., M.X.X, W.H. collected 
and analyzed the raw data. Z.G.D., T.X.G. and S.M.Z. wrote the manuscript. X.L., C.D.D., K.D.L.

revised the manuscript. All authors read and approved the final manuscript

Conflict of Interest: The authors declare no conflict of interest.

\section{Reference}

1 Wang, C., Horby, P. W., Hayden, F. G. \& Gao, G. F. A novel coronavirus outbreak of global health concern. Lancet 395, 470-473, doi:10.1016/S0140-6736(20)30185-9 (2020).

2 Wu, F. et al. A new coronavirus associated with human respiratory disease in China. Nature 579, 265-269, doi:10.1038/s41586-020-2008-3 (2020).

3 Zhu, N. et al. A Novel Coronavirus from Patients with Pneumonia in China, 2019. N Engl J Med 382, 727-733, doi:10.1056/NEJMoa2001017 (2020).

4 Lu, R. et al. Genomic characterisation and epidemiology of 2019 novel coronavirus: implications for virus origins and receptor binding. Lancet 395, 565-574, doi:10.1016/S0140-6736(20)30251-8 (2020).

5 Selvan, M. E. Risk factors for death from COVID-19. Nature reviews. Immunology, doi:10.1038/s41577-020-0351-0 (2020).

6 An, P. J., Yi, Z. Z. \& Yang, L. P. Biochemical indicators of coronavirus disease 2019 exacerbation and the clinical implications. Pharmacol Res, 104946, doi:10.1016/j.phrs.2020.104946 (2020).

$7 \quad \mathrm{Xu}, \mathrm{Z}$. et al. Pathological findings of COVID-19 associated with acute respiratory distress syndrome. Lancet Respir Med 8, 420-422, 
doi:10.1016/S2213-2600(20)30076-X (2020).

8 Oberfeld, B. et al. SnapShot: COVID-19. Cell 181, 954-954 e951, doi:10.1016/j.cell.2020.04.013 (2020).

9 Qin, C. et al. Dysregulation of immune response in patients with COVID-19 in Wuhan, China. Clinical infectious diseases : an official publication of the Infectious Diseases Society of America, doi:10.1093/cid/ciaa248 (2020).

10 Tan, M. et al. Immunopathological characteristics of coronavirus disease 2019 cases in Guangzhou, China. Immunology, doi:10.1111/imm.13223 (2020). Pathophysiology, Animal Models, and Anti-Viral Interventions. Pathogens 9, doi:10.3390/pathogens9060426 (2020).

12 Sun, S. H. et al. A Mouse Model of SARS-CoV-2 Infection and Pathogenesis. Cell Host Microbe, doi:10.1016/j.chom.2020.05.020 (2020).

13 Boe, D. M., Curtis, B. J., Chen, M. M., Ippolito, J. A. \& Kovacs, E. J. Extracellular traps and macrophages: new roles for the versatile phagocyte. Journal of leukocyte biology 97, 1023-1035 (2015).

14 Liu, S. et al. Neutrophil extracellular traps are indirectly triggered by lipopolysaccharide and contribute to acute lung injury. Scientific reports 6,37252 (2016).

15 Narasaraju, T. et al. Excessive neutrophils and neutrophil extracellular traps contribute to acute lung injury of influenza pneumonitis. The American journal of pathology 179 , 199-210 (2011). 
16 Lefrançais, E., Mallavia, B., Zhuo, H., Calfee, C. S. \& Looney, M. R. Maladaptive role of neutrophil extracellular traps in pathogen-induced lung injury. JC/ insight 3 (2018).

17 Zhao, X. et al. Neutrophils undergo switch of apoptosis to NETosis during murine fatty liver injury via S1P receptor 2 signaling. Cell Death \& Disease 11, 1-14 (2020).

18 Pulavendran, S. et al. Production of Neutrophil Extracellular Traps Contributes to the Pathogenesis of Francisella tularemia. Frontiers in immunology 11, 679, doi:10.3389/fimmu.2020.00679 (2020).

19 Wang, Y. et al. Remdesivir in adults with severe COVID-19: a randomised, double-blind, placebo-controlled, multicentre trial. The Lancet (2020).

20 Molina, J. M. et al. No evidence of rapid antiviral clearance or clinical benefit with the combination of hydroxychloroquine and azithromycin in patients with severe COVID-19 infection. Med Mal Infect 10 (2020).

21 Law, H. K. et al. Toll-like receptors, chemokine receptors and death receptor ligands responses in SARS coronavirus infected human monocyte derived dendritic cells. BMC immunology 10, 35 (2009).

22 Vercammen, E., Staal, J. \& Beyaert, R. Sensing of viral infection and activation of innate immunity by toll-like receptor 3. Clinical microbiology reviews 21, 13-25 (2008).

23 Enjuanes, L. et al. Molecular Basis of Coronavirus Virulence and Vaccine Development. Advances in virus research 96, 245-286, doi:10.1016/bs.aivir.2016.08.003 (2016).

24 Mazaleuskaya, L., Veltrop, R., Ikpeze, N., Martin-Garcia, J. \& Navas-Martin, S. Protective role of Toll-like Receptor 3-induced type I interferon in murine coronavirus 
infection of macrophages. Viruses 4, 901-923, doi:10.3390/v4050901 (2012).

25 Totura, A. L. et al. Toll-Like Receptor 3 Signaling via TRIF Contributes to a Protective Innate Immune Response to Severe Acute Respiratory Syndrome Coronavirus Infection. mBio 6, e00638-00615, doi:10.1128/mBio.00638-15 (2015).

26 Cao, X. COVID-19: immunopathology and its implications for therapy. Nature reviews. Immunology 20, 269-270, doi:10.1038/s41577-020-0308-3 (2020).

27 Zhang, B. et al. Immune phenotyping based on neutrophil-to-lymphocyte ratio and IgG predicts disease severity and outcome for patients with COVID-19. medRxiv (2020).

28 Chen, X. et al. Restoration of leukomonocyte counts is associated with viral clearance in COVID-19 hospitalized patients. MedRxiv (2020).

29 Zheng, M. et al. Functional exhaustion of antiviral lymphocytes in COVID-19 patients. Cellular \& molecular immunology, 1-3 (2020).

30 Channappanavar, R. \& Perlman, S. Pathogenic human coronavirus infections: causes and consequences of cytokine storm and immunopathology. Seminars in immunopathology 39, 529-539, doi:10.1007/s00281-017-0629-x (2017).

31 Kang, S., Tanaka, T., Narazaki, M. \& Kishimoto, T. Targeting interleukin-6 signaling in clinic. Immunity 50, 1007-1023 (2019).

32 Cao, X. COVID-19: immunopathology and its implications for therapy. Nature reviews immunology 20, 269-270 (2020).

33 Liao, M. et al. Single-cell landscape of bronchoalveolar immune cells in patients with COVID-19. Nature Medicine, 1-3 (2020).

34 Ahmadpoor, P. \& Rostaing, L. Why the immune system fails to mount an adaptive 
bioRxiv preprint doi: https://doi.org/10.1101/2020.06.07.119032; this version posted July 2, 2020. The copyright holder for this preprint (which was not certified by peer review) is the author/funder, who has granted bioRxiv a license to display the preprint in perpetuity. It is made available under aCC-BY-NC 4.0 International license.

immune response to a Covid-19 infection. Transplant International(2020).

35 Antonelou, M. et al. Therapeutic Myeloperoxidase Inhibition Attenuates Neutrophil Activation, ANCA-Mediated Endothelial Damage, and Crescentic GN. Journal of the American Society of Nephrology : JASN 31, 350-364, doi:10.1681/ASN.2019060618 (2020).

36 Okeke, E. B. et al. Inhibition of neutrophil elastase prevents neutrophil extracellular trap formation and rescues mice from endotoxic shock. Biomaterials 238, 119836, doi:10.1016/j.biomaterials.2020.119836 (2020). 
Figure 1: SARS-CoV-2 mimic induced acute lung injury in BALB/c mice. (a) A schematic description of Poly I:C and SARS-CoV-2 spike protein intratracheal administration in mice. (b) Histological characteristics of lung injury and interstitial pneumonia induced by Poly I:C and SARS-CoV-2 spike protein (Poly I:C + SP); 6 h, 24 h and 48 h after challenge. Control group; Saline and Poly I:C $24 \mathrm{~h}$ challenge. Black scale bar $=100 \mu \mathrm{m}$, blue scale bar $=50 \mu \mathrm{m}$. (c) In vivo small animal MRI documented pleural effusion induced by Poly I:C and SARS-CoV-2 spike protein; 6 h, 24 h and $48 \mathrm{~h}$ after challenge. Control group; Saline and Poly I:C $24 \mathrm{~h}$ challenge.

Figure 2: Poly I:C plus SP induced cytokines release storm in lung. (a) Production of IL-6 in the BAL from Poly I:C plus SP challenged mice, ${ }^{* *} P<0.01, * * * P<0.001$. (b) Levels of dsDNA in the BAL from Poly I:C plus SP challenged mice, $* * * P<0.001$. (c) Total cell count in the BAL from Poly I:C and $5 \mu \mathrm{g}, 10 \mu \mathrm{g}$ or $15 \mu \mathrm{g}$ SARS-CoV-2 spike challenged mice, control group; Saline. $* P<0.05$, ** $P$ $<0.01, * * * P<0.001 .6 \mathrm{~h}$ or $24 \mathrm{~h}$ after SARS-CoV-2 mimic challenged mice. Control group; Saline (d) Cellular composition in BAL, * $P<0.05$. (e) BAL IL-6 concentration. $* P<0.05$, ** $P<0.01$, *** $P<$ 0.001. (f) Cellular composition in BAL, ** $P<0.01$, *** $P<0.001$. (g) Levels of dsDNA in the BAL. $* P<0.05, * * * P<0.001$. Single cells dissociated from Poly I:C plus SP challenged mice lung (h) IL-6 concentration from cell culture medium, $* * * P<0.001$.

Figure 3: Blocking of inflammatory cytokines. BAL IL-6, IL-1 $\alpha$ and TNF $\alpha$ concentrations from anti-IL-6 mAbs (a), anti-IL-6R mAbs (b), anti-TNF $\alpha$ mAbs (c), anti-TNFR2 mAbs (d), anti-IL-1 $\alpha$ mAbs (e), anti-GM-CSF (f) mAbs treated mice. $* P<0.05$, ** $P<0.01$. Cellular composition in BAL, anti-IL-6 (g), anti- IL-6R (h), anti-TNF $\alpha$ (i), anti-TNFR2 (j), anti-IL-1 $\alpha$ (k) or anti-GM-CSF (l) mAbs treated mice. (m) Levels of dsDNA in the BAL. Poly I:C stimulate the IL-6 production in mouse macrophages (n) IL-6 concentration from cell culture medium. (o) Histological characteristics of lung injury and interstitial pneumonia. Black scale bar $=100 \mu \mathrm{m}$, blue scale bar $=50 \mu \mathrm{m}$

Figure 4: Blocking of inflammatory related signaling pathways. (a) BAL IL-6, IL-1 $\alpha$ and TNF $\alpha$ concentrations. $* P<0.05$. (b) Percent of neutrophils in BAL. $* P<0.05$. (c) Levels of dsDNA in the BAL. Poly I:C stimulate the IL-6 production in mouse primary macrophages (d) IL-6 concentration from cell culture medium. $* P<0.05$. (e) Histological characteristics of lung injury and interstitial pneumonia. Black scale bar $=100 \mu \mathrm{m}$, blue scale bar $=50 \mu \mathrm{m}$.

Figure 5: Graphical abstract of SARS-CoV-2 mimic and applications of therapeutic mAbs and inhibitors. 

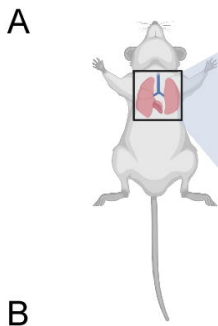

Saline $24 \mathrm{~h}$

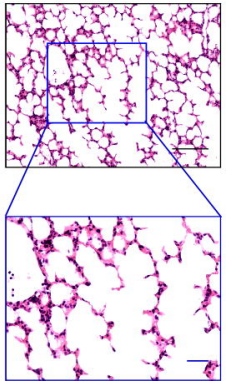

C

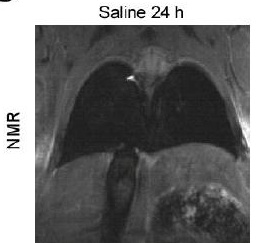

Intratracheal instillation

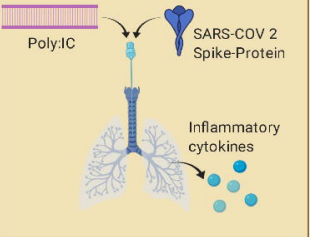

Poly I:C $24 \mathrm{~h}$

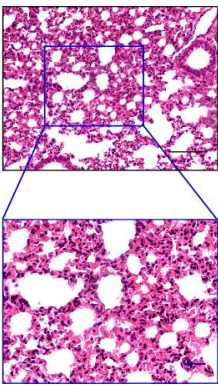

Poly I:C+SP 6 h

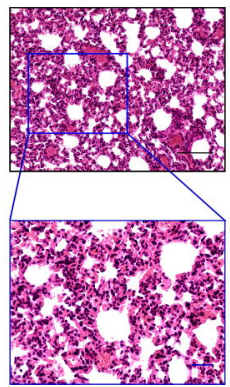

Poly I:C+SP $24 \mathrm{~h}$

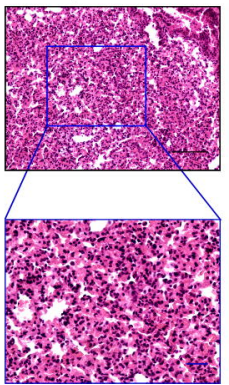

Poly I:C+SP 48 h

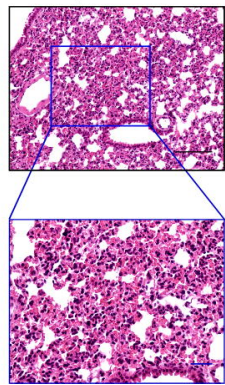

Poly I:C $24 \mathrm{~h}$

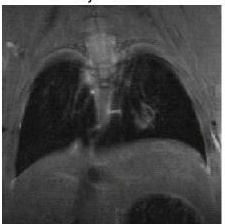

Poly I:C+SP 6 h

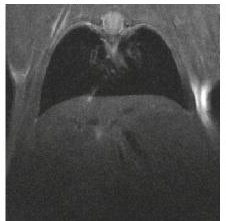

Poly l:C+SP 24 h

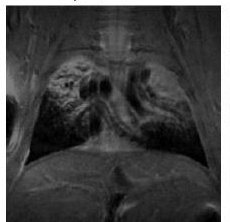

Poly I:C+SP 48 h

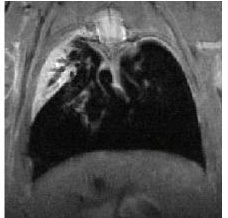




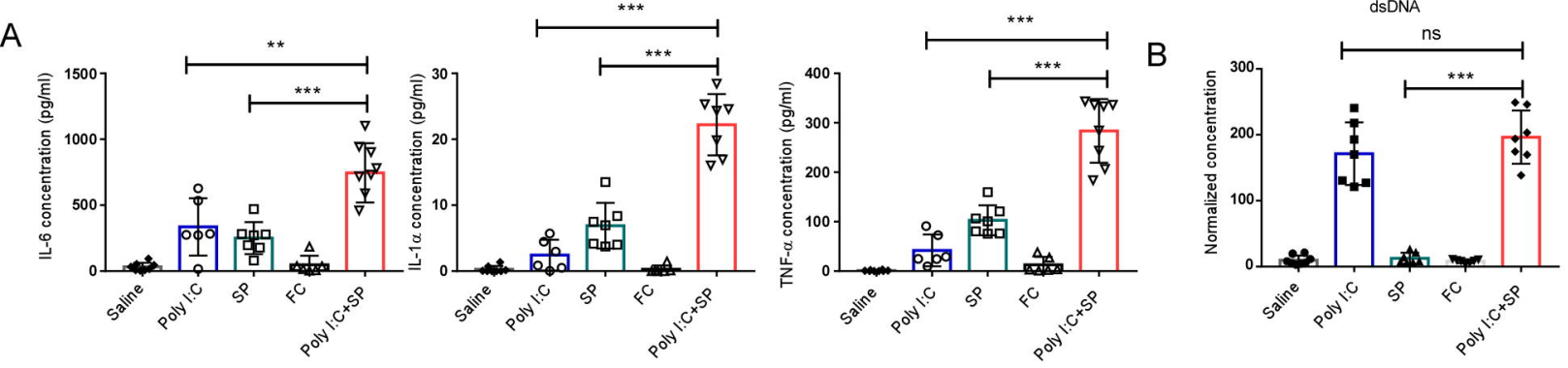

C

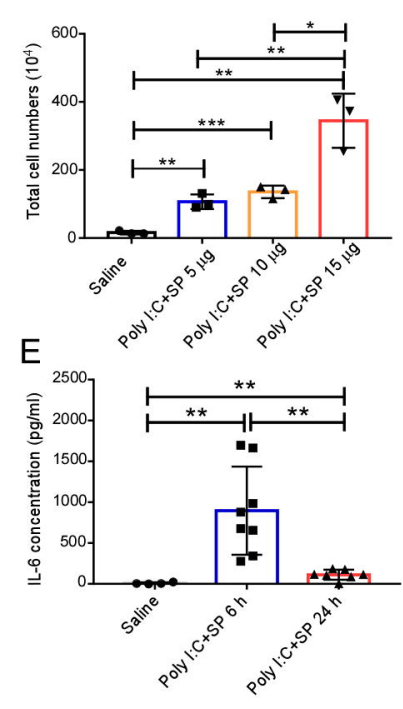

F

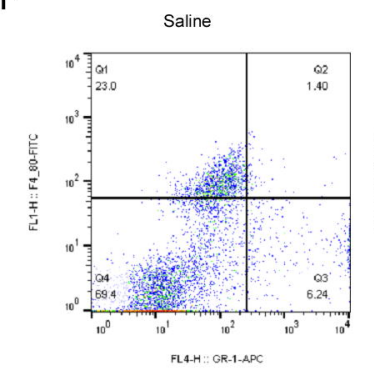

D
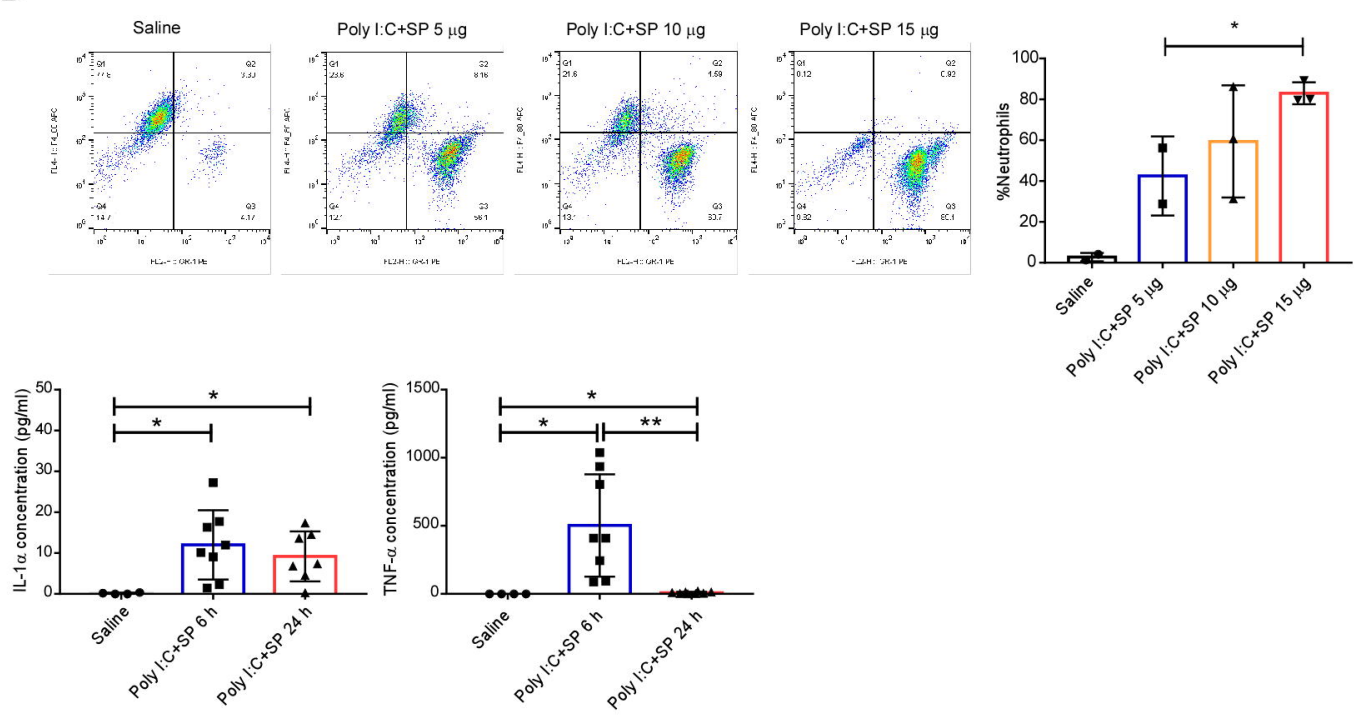

Poly l:C+SP $6 \mathrm{~h}$

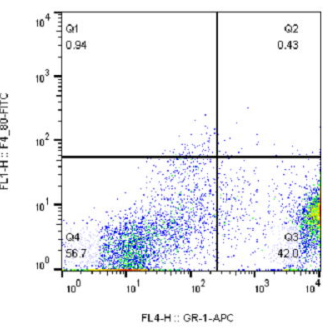

Poly I:C+SP 24 h

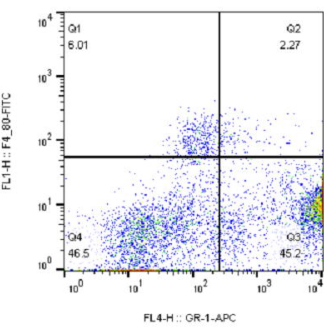

G

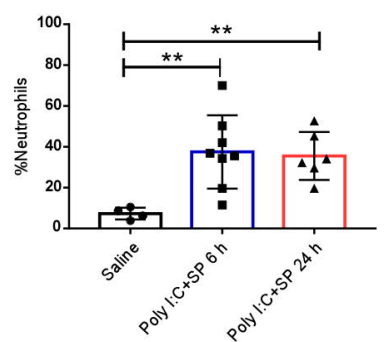

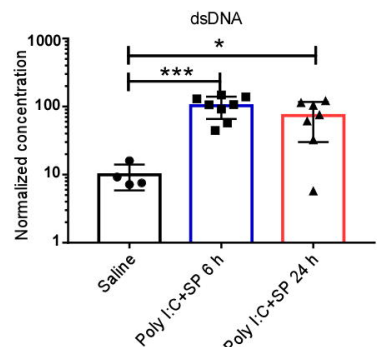

$\mathrm{H}$

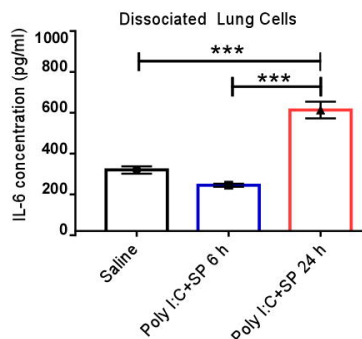



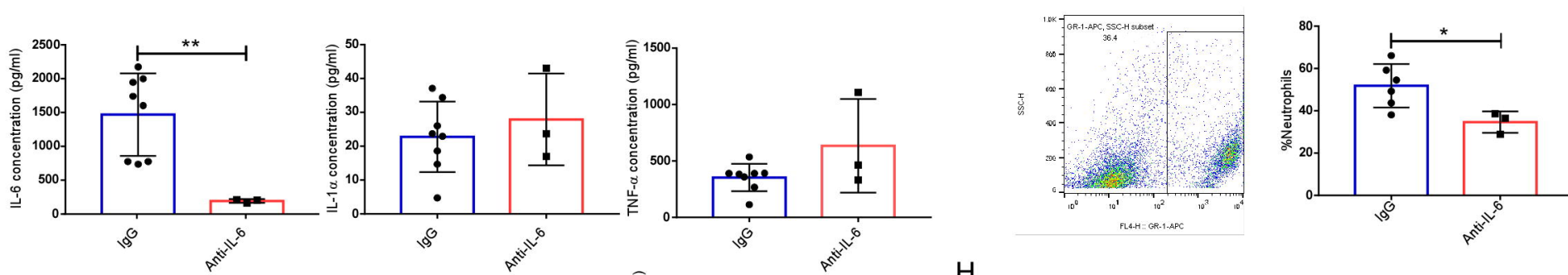

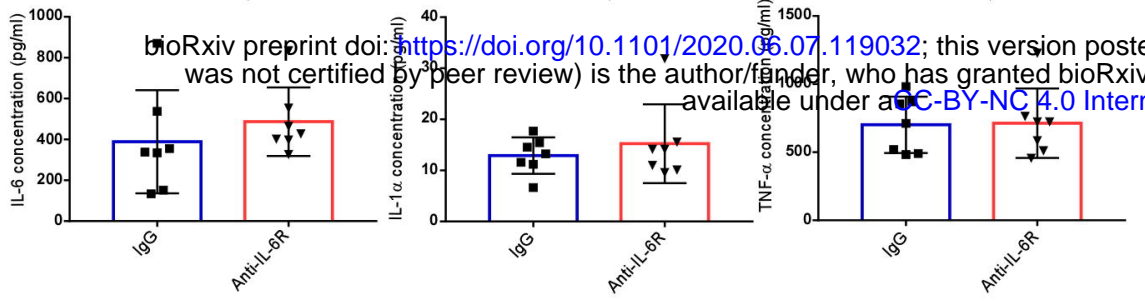

$\mathrm{H}$

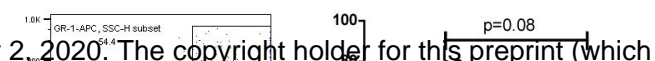
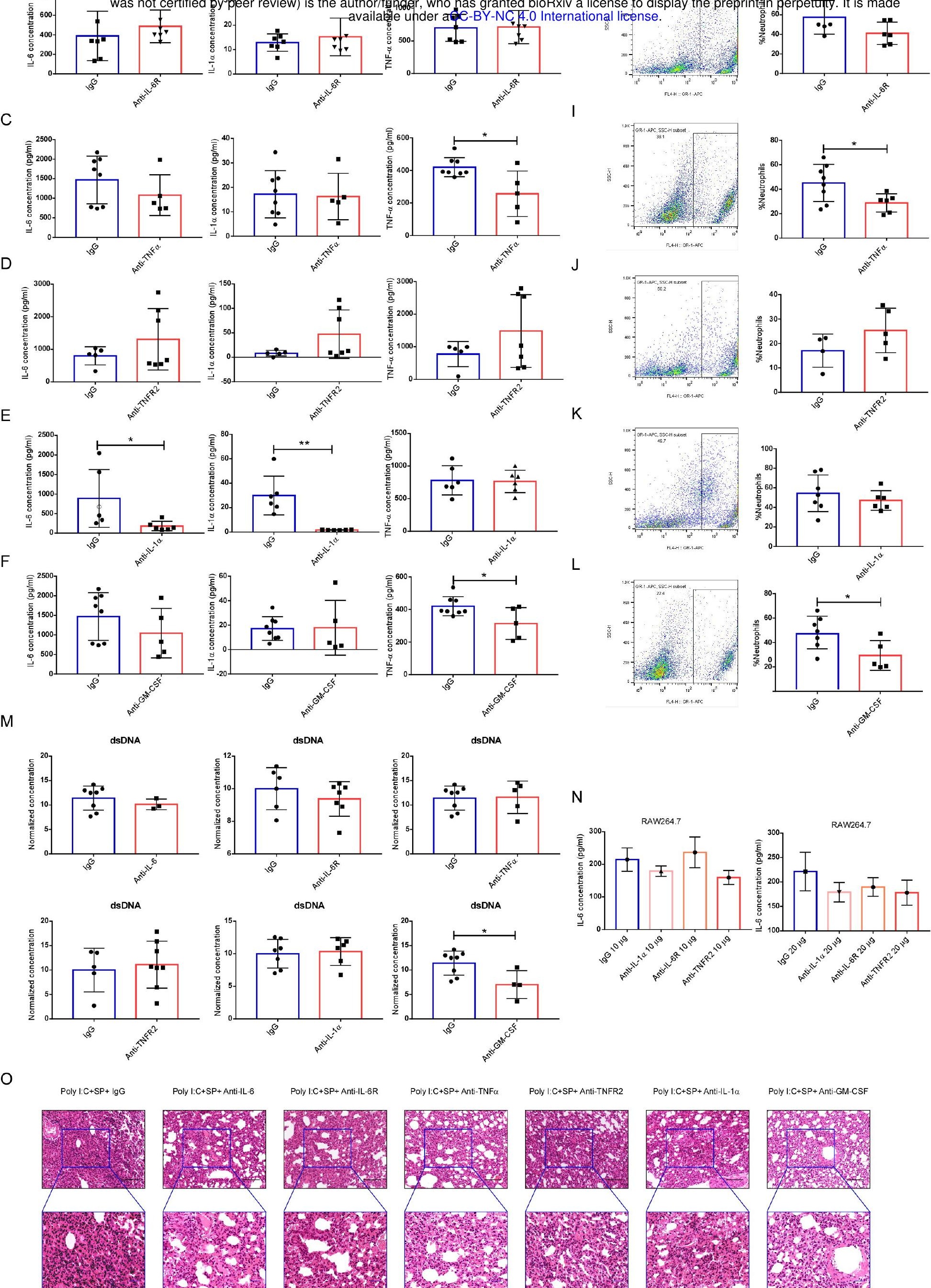


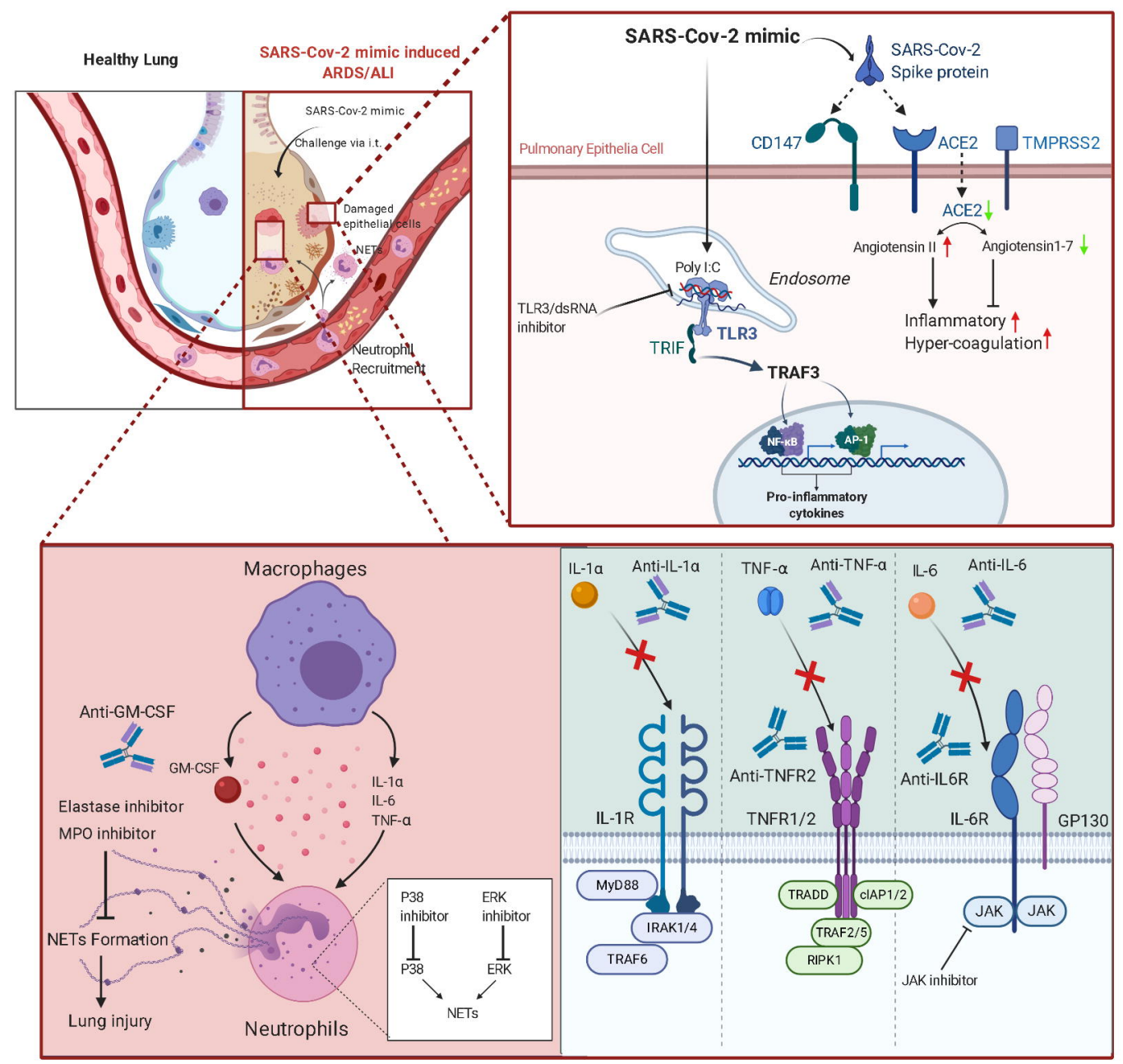

